\title{
Balzac, Nouvelles et contes I (1820-1832), 2005 ; Nouvelles et contes II (1832-1850), 2006
}

\section{Nicole Mozet}

\section{(2) OpenEdition}

1 Journals

\section{Édition électronique}

URL : http://journals.openedition.org/genesis/406

DOI : $10.4000 /$ genesis.406

ISSN : 2268-1590

Éditeur :

Presses universitaires de Paris Sorbonne (PUPS), Société internationale de génétique artistique littéraire et scientifique (SIGALES)

Édition imprimée

Date de publication : 10 octobre 2010

Pagination : 161-162

ISBN : 978-2-84050-711-6

ISSN : 1167-5101

\section{Référence électronique}




\section{Comptes rendus d'ouvrages}

Nouvelles et contes I (1820-1832), Balzac, Gallimard, coll. «Quarto », 2005, 1763 p. ; Nouvelles et contes II (1832-1850), Gallimard, coll. «Quarto », 2006, 1873 p., édition établie, présentée et annotée par Isabelle Tournier.

\section{Compte rendu par Nicole Mozet}

Deux gros volumes pour l'édition exhaustive des textes brefs de Balzac en suivant l'ordre de la première publication, soit en revue ou dans un journal, soit en librairie. Une édition génétique originale, sans les manuscrits ni la version finale. Balzac, qui a toute sa vie réédité ses œuvres en les remaniant - ce qui revenait à transformer de l'imprimé en manuscrit avant de l'envoyer à l'imprimerie -, se prêtait admirablement à cette expérimentation. Ce que la critique balzacienne appelle édition originale ${ }^{1}$ de La Comédie humaine (dite «édition Furne $\left.{ }^{2} »\right)$ est en réalité la dernière édition des romans et nouvelles de Balzac, dont beaucoup remontent à dix ou quinze ans. Tous d'ailleurs n'ont pas été repris dans La Comédie humaine. En outre, lorsque l'auteur est mort en 1850, il a laissé un grand nombre de corrections sur son exemplaire personnel de La Comédie humaine, dit «Furne corrigé », qui sert de base à la plupart des éditions modernes des textes balzaciens, à commencer par celle de la collection de la Pléiade. C'est tout à l'honneur des éditions Gallimard d'avoir considéré que ces deux entreprises, loin d'être contradictoires, méritaient d'être accueillies chacune dans une collection de la maison. Ainsi Isabelle Tournier s'est-elle faufilée sur un chemin escarpé entre deux massifs considérables qu'elle a volontairement laissés de côté : les brouillons et épreuves corrigées en amont, les romans et leurs avatars de l'autre côté. La grande période $\mathrm{du}$ « contier » en ressort évidemment plus lumineuse, elle occupe en fait tout le premier volume et un large début du second. Ce qui est visé, dans une sorte de recherche de l'Absolu, c'est ce que Dai Sijie, l'auteur de Balzac et la petite tailleuse chinoise, appelle du «Balzac tout pur $^{3}$ », le noyau autour duquel gravite tout le reste. Ce n'est ni un brouillon ni une ébauche, quelquefois un fragment. Jamais un roman, qui dans cette perspective devient en quelque sorte le tombeau de la nouvelle qui lui a servi d'embryon. Toute la moderne écriture romanesque de la fin de la Restauration et du début de la monarchie de Juillet a usé de la nouvelle comme d'un tremplin. Nous en avons maints témoignages, comme ici ce commentaire de Balzac à propos du Père Goriot, « qui est devenu sous mes doigts un livre aussi considérable que l'est Eugénie Grandet ou Ferragus ${ }^{4}$ ». De même que les stratégies balzaciennes de publication relèvent pour une part de «montages », telles les mises en séries des différentes « Scènes de la vie... » ou La Comédie humaine elle-même, de même l'ouvrage d'Isabelle Tournier manifeste-t-il sa fidélité à Balzac en adoptant lui aussi la technique du montage : il nous restitue la chronologie de l'écriture, son historicité. C'est donc bien d'une édition génétique qu'il s'agit, pas d'une simple anthologie.

Prenons l'exemple de la nouvelle La Grande Bretèche, l'un des textes les plus vagabonds de La Comédie humaine, dont le titre (qui figure sur le manuscrit) est présent ou non selon les modes de publication. Prévue pour le volume collectif des Contes bruns (1832), c'est en fait dans le tome III des Scènes de la vie privée, couplée avec Le Message et sans titre, que la nouvelle est publiée pour la première fois : cette version figure donc à sa date dans le premier volume, p. 14011420. Ce diptyque, intitulé Le Conseil, ne sera jamais repris, et le divorce demeurera radical entre Le Message et La Grande Bretèche. On retrouve le texte en 1837 dans le second volume, avec un tout autre montage, sous le titre La Grande Bretèche ou les Trois Vengeances. La nouvelle est cette fois regroupée avec trois autres nouvelles venues des Contes bruns et sera rééditée en 1839. En 1845, dans l'édition Furne de La Comédie humaine, elle figure seule pour la première fois, sous le titre : La Grande Bretèche (Fin de Autre Étude de femme ${ }^{5}$ ). Le titre a été rayé par l'auteur, qui a ajouté cette indication manuscrite : "Ceci doit être reporté à la fin de Autre Étude de femme ». La Grande Bretèche devra donc perdre à nouveau son titre pour aller rejoindre d'autres textes issus des Contes bruns de 1832. Ce retour aux origines dit bien la complexité du temps balzacien de l'écriture, temps en spirale qui avance par bonds, avec maints retours, arrêts, dérivations et reprises.

1. Au sens classique : première publication légale en librairie.

2. À partir de 1842, dix-sept volumes du vivant de Balzac. Ce texte est disponible en ligne sur le site de la Maison de Balzac à Paris, qui a repris et corrigé la saisie effectuée par le Groupe international de recherches balzaciennes pour le CD-Rom « Explorer La Comédie humaine » (1999).

3. II, p. 1581-1594.

4. II, p. 1626, lettre du 22 juillet 1834 à l'imprimeur Éverat (Correspondance, II, p. 560). 5. II, p. 1302-1334. 
Pour guider son lecteur dans ce parcours chronologique et en faire ressortir la dimension génétique, Isabelle Tournier a mis en place plusieurs sortes de panneaux indicateurs. Chaque texte est précédé d'une brève introduction. À la fin de chaque volume, on trouve les « histoires des textes » (I, p. 1717-1753; II, p. 1807-1851) et une très copieuse biographie de l'écrivain, sous le titre «Vie et œuvre » (I, p. 16111716 ; II, p. 1595-1806), accompagnée d'une belle iconographie. À l'intérieur des volumes, chaque périodisation (1820-mars 1830 ; avril 1830 ; avril 1830-septembre 1831 , etc.) est précédée d'une mise au point d'une dizaine de pages : « Les ateliers d'un clandestin », «L'entrée en scène », «L'admirable kaléidoscope », etc.). La biographie, année après année et jour après jour, suit elle aussi un strict principe chronologique. La perspective génétique sert de fil directeur, car la question première demeure toujours celle des conditions qui permettent ou entravent la venue à l'écriture et surtout le passage à la publication. Étayée par les mises au point qui font synthèse et les histoires des textes, cette biographie est le filet qui tient l'ensemble. Cette organisation relève elle aussi d'un subtil «montage ».

Il y a là un choix éditorial à propos duquel on peut véritablement parler d'« amour des commencements » (expression que j'emprunte à J.-B. Pontalis). À traquer non pas le premier jet (souvent laborieux chez Balzac), mais l'explosion libératrice de la première publication, surtout pour le jeune écrivain, pas encore enchaîné dans l'engrenage de l'écriture sans fin, on rencontre quelque chose qui est de 1'ordre de la jouissance. Isabelle Tournier réussit à nous communiquer ce qu'elle appelle une certaine «contagion » de la joie :

À les lire [ces premiers textes], enlevés, portés par leur intensité comique, dramatique, funèbre, leur esprit, reposant tout entiers sur une idée, une situation, presque rien, on ressent la contagion de la joie que Balzac eut à les écrire (I, p. 10).
Le second volume (1832-1850), à l'arrière-plan duquel se profilent les grands feuilletons et les tentatives théâtrales, se caractérise par les expérimentations sur les genres - entre journalisme et fiction - et les supports d'écriture. Souvent, comme pour La Muse du département, les articles $\mathrm{du}$ journaliste seront repris par le romancier. C'est une autre façon de répéter sans ressasser.

Avec ces deux volumes, l'édition balzacienne ne s'est pas seulement enrichie d'une nouvelle publication offrant à la lecture beaucoup de textes difficilement accessibles. Nous sommes immédiatement amenés à partager une réflexion qui se déploie sur trois axes parallèles : qu'est-ce qu'une forme brève ? Que nous apprennent, entre manuscrit et remaniements postérieurs, les premières publications ? Comment transformer la chronologie en outil génétique ? Quoique spécialement adaptées à Balzac, ces questions portent plus généralement sur le rapport de l'écrivain aux dates de son temps : naître en 1799, se marier en 1850 au même âge que son père, mais pour mourir quelques mois plus tard, publier l'essentiel d'une grande œuvre romanesque entre 1830 et 1848 , se lancer ensuite au théâtre... Quel est l'impact des révolutions sur l'écriture? Pourquoi La Peau de chagrin (roman) en 1831 et La Marâtre (théâtre) en 1848 ? Histoire et génétique se croisent et se confortent.

Bilder der Handschrift. Die graphische Dimension der Literatur, dir. Davide Giuriato, Stephan Kammer, Frankfurt am Main, Stroemfeld/Nexus, 2006, 296 p.

\section{Compte rendu par Catherine Viollet}

La couverture noire reproduisant en blanc une note de Walter Benjamin intitulée « Sur la perception en soi » présente avec l'élégance coutumière du Stroemfeld Verlag le contenu du volume intitulé Bilder der Handschrift (Images de l'écriture manuscrite). Les neuf contributions rassemblées, de manière remarquablement homogène, par Davide Giuriato et Stephan Kammer visent avant tout à convaincre de l'intérêt d'observer et de prendre en compte la dimension matérielle et graphique de l'écriture littéraire.

Certes, la question n'est pas tout à fait nouvelle, et ces contributions s'appuient sur une abondante bibliographie, principalement de langue allemande (Medienwissenschaft, Editionswissenschaft), mais s'inspirent aussi des textual studies, des écrits de Barthes, Derrida, Deleuze et Guattari entre autres, ainsi que de la critique génétique française. La portée du propos tient principalement au fait que les questionnements théoriques et méthodologiques de l'ouvrage sont systématiquement associés à une démonstration concrète.

Les corpus étudiés, qui s'étendent sur deux siècles (1770-1980), ont en commun d'être de taille restreinte, ce qui facilite la démonstration, et de se répondre mutuellement dans l'analyse des dispositifs en jeu. Ils permettent d'aborder divers aspects de la relation entre écrit (Schrift) et processus d'écriture (Schreiben) : interactions entre l'écriture et la voix, le dessin, les dispositifs graphiques et spatiaux, stratégies ambivalentes, questions de reproductibilité, mises en scène de l'acte scriptural. Certaines des contributions concernent également des aspects que l'on pourrait désigner par l'expression de « métagénétique », c'est-à-dire lorsque le processus de genèse constitue l'objet même du document manuscrit étudié.

Un tel projet remet d'emblée en question les présupposés traditionnels de la critique littéraire, où le texte est généralement considéré indépendamment des aspects concrets, matériels de son élaboration. Il s'inscrit dans une théorie esthétique qui tend à cerner l'œuvre littéraire de manière globale, où les aspects matériels et sensibles de la « scène de l'écriture » se montrent indissociables de la construction du sens textuel, renouvelant ainsi les modalités de perception et de lecture. Les documents autographes du processus 